King's College/KCL-TH-97-30

hep-th/9707184

August 27, 2018

\title{
Weyl Superspace
}

\author{
P.S. Howe, \\ Dept. of Mathematics, \\ King's College, London, UK.
}

\begin{abstract}
It is shown that the equations of motion of eleven-dimensional supergravity follow from setting the dimension zero components of the superspace torsion tensor equal to the Dirac matrices. The proof of this assertion is facilitated by the introduction of a connection taking its values in the Lie algebra of $C \operatorname{Spin}(1,10)=\operatorname{Spin}(1,10) \times \mathbb{R}^{+}$. The resulting formulation of eleven-dimensional supergravity is Weyl covariant but is equivalent to the usual formulation modulo topological considerations.
\end{abstract}


In view of the current interest in eleven-dimensional supergravity it is useful to try to gain a better understanding of the superspace geometry of the theory. In this note we show that this geometry is very tightly constrained to the extent that the standard choice for the dimension zero torsion is actually sufficient to put the theory on shell. This may be useful, for example, from the point of view of trying to find higher order corrections to the theory which are expected to be present in the conjectured $M$-theory. The analysis of the consequences of the basic constraint is simplified by the introduction of a connection which takes its values in the Lie algebra of $C \operatorname{Spin}(1,10)=\operatorname{Spin}(1,10) \times \mathbb{R}^{+}$, and we call a superspace with such a connection Weyl Superspace. The formulation of supergravity we shall arrive at is therefore locally scale invariant, but in a somewhat trivial way. The associated scale curvature tensor vanishes and so one can recover the usual theory in a suitable gauge, at least locally.

The equations of motion of eleven-dimensional supergravity [1] were presented in the superspace formalism some time ago [2, 3], and the constraints have been interpreted in terms of membranes [4, 5] and as integrability conditions in membrane superspace [6]. More recently, the authors of ref. [7] carried out an analysis of the geometrical superspace constraints (i.e. without including the four-form field strength initially) and showed that the standard dimension zero torsion constraint implies that, at dimension one-half, there is a single spinor field appearing in the torsion. They then set this field to zero and recovered the standard on-shell formalism. In this note we ask what happens if one does not set this spinor field to zero. It turns out, ignoring topological niceties, that it can be written as the derivative of a scalar superfield and that this scalar superfield can be transformed away by a super-Weyl transformation. It is, however, easier to demonstrate this by modifying the formalism slightly to include a scale connection in addition to the usual Lorentz connection. In fact, the result implies that we can summarise the equations of motion of supergravity in eleven dimensions without introducing a connection at all, and we start off with a brief discussion of this before reverting to the connection formalism to prove the main point.

The basic structure we shall study can be called a special superconformal structure in (11|32)dimensional superspace $M$. Such a structure is a choice of odd tangent bundle $F$ having rank $(0 \mid 32)$ with associated Frobenius tensor which is maximally non-integrable and invariant under $C \operatorname{Spin}(1,10):=\operatorname{Spin}(1,10) \times \mathbb{R}^{+}$. The Frobenius tensor is defined as follows: for any two odd vector fields $X, Y$ (i.e. sections of $F$ ) one computes their Lie bracket and evaluates it modulo $F$. This defines, at each point $p \in M$, a map $\wedge^{2} F_{p} \rightarrow B_{p}=T_{p} / F_{p}$, and hence a section of $\wedge^{2} F^{*} \otimes B$. The requirement of $C \operatorname{Spin}(1,10)$ invariance means that one can choose bases $\left\{E_{\alpha}\right\},\left\{E^{a}\right\}$ for $F$ and $B^{*}$ respectively such that the components of the Frobenius tensor in such a basis, denoted by $\hat{T}_{\alpha \beta}{ }^{c}$, and by definition given by

$$
\hat{T}_{\alpha \beta}^{c}=-\left\langle\left[E_{\alpha}, E_{\beta}\right], E^{c}\right\rangle,
$$

where $\langle$,$\rangle denotes the usual pairing between vectors and forms, are proportional to the Dirac$ matrices,

$$
\hat{T}_{\alpha \beta}^{c}=-i\left(\Gamma^{c}\right)_{\alpha \beta}
$$

Note that, although we have used the usual torsion notation here, no connection has been introduced as yet. Note also that the Dirac matrices define a tensor which is $\operatorname{CSpin}(1,10)$ invariant and not just $\operatorname{Spin}(1,10)$ invariant.

For dimensions $D<11$ it is necessary to supplement this basic conformal constraint with further constraints in order to obtain Poincaré supergavity, but in $D=11$ this is not the case. In fact we can show that a special superconformal structure on an (11|32)-dimensional superspace, $M$, 
is equivalent to the equations of motion of eleven-dimensional supergravity, modulo toplogical considerations.

To prove this one makes a choice of even tangent bundle $B$ (so that $T=F \oplus B$ ) and introduces a suitable connection, $\hat{\Omega}$, which takes its values in the Lie algebra of $C \operatorname{Spin}(1,10)$. Thus one can write

$$
\begin{aligned}
\hat{\Omega}_{\alpha}{ }^{\beta} & =\Omega_{\alpha}^{\prime \beta}+\delta_{\alpha}{ }^{\beta} K \\
\hat{\Omega}_{a}{ }^{b} & =\Omega_{a}^{\prime b}+2 \delta_{a}{ }^{b} K
\end{aligned}
$$

where $K$ is the scale connection one-form, $\Omega_{a b}^{\prime}=-\Omega_{b a}^{\prime}$ is the $\mathfrak{s p i n}(1,10)$ connection and

$$
\Omega_{\alpha}^{\prime \beta}=\frac{1}{4}\left(\Gamma_{a b}\right)_{\alpha}^{\beta} \Omega_{a b}^{\prime}
$$

The associated curvatures will be denoted $\hat{R}, R^{\prime}$ and $G$, the latter being the scale curvature. The convention we shall use is that spinor (vector) indices are lowered or raised with $\eta_{\alpha \beta}\left(\eta_{a b}\right)$ and their inverses, written with upper indices, where $\eta_{\alpha \beta}$ is the charge conjugation matrix and is antisymmetric and $\eta_{a b}$ is the usual Lorentz metric. These operations are not covariant with respect to scale transformations so that one has to keep track of the indices which have been raised or lowered in this way. We shall also write the torsion with a hat in Weyl superspace as we shall later transform back to Lorentzian superspace.

By a suitable choice of $B$ (in other words of $E_{a}$ ) and of the spinorial part of the connection one can arrange that the dimension one-half components of the torsion tensor, $\hat{T}_{\alpha \beta}{ }^{\gamma}$ and $\hat{T}_{\alpha b}{ }^{c}$, vanish. At dimension one we may choose the vectorial components of the connections such that

$$
\hat{T}_{a b}{ }^{c}=0 ; \quad\left(\Gamma_{a}\right)^{\alpha \beta} G_{\alpha \beta}=0 .
$$

The Bianchi identities then imply that 1

$$
\hat{T}_{a \beta}^{\gamma}=-\frac{1}{36}\left(\Gamma^{b c d}\right)_{\beta}^{\gamma} H_{a b c d}-\frac{1}{288}\left(\Gamma_{a b c d e}\right)_{\beta}{ }^{\gamma} H^{b c d e}
$$

as well as

$$
G_{\alpha \beta}=0,
$$

where $H_{a b c d}$ is totally antisymmetric and has Weyl weight 2. For completeness we give the curvature tensor at dimension one:

$$
R_{\alpha \beta, a b}^{\prime}=\frac{1}{6}\left(\left(\Gamma^{c d}\right)_{\alpha \beta} H_{a b c d}+\frac{1}{3}\left(\Gamma_{a b c d e f}\right)_{\alpha \beta} H^{c d e f}\right) .
$$

It is now a simple matter to prove that $G=0$ by using the Bianchi identity for the scale curvature, $d G=0$. At dimension three-halves this identity gives, since the dimension one component of $G$ vanishes,

$$
\hat{T}_{(\alpha \beta}{ }^{c} G_{\gamma) c}=0
$$

which implies that $G_{\alpha b}=0$. At dimension two one then has

$$
\hat{T}_{\alpha \beta}{ }^{c} G_{c d}=0 \Rightarrow G_{c d}=0 .
$$

\footnotetext{
${ }^{1} H$ in this paper differs by a sign to that of [3].
} 
The analysis of the remaining dimension three-halves and two Bianchi identities is then the same as in the original papers except that the covariant derivatives which arise include the scale connection $K$. At dimension three-halves one finds that the dimension three-halves torsion is expressible as a derivative of $H$ :

$$
\hat{T}_{a b}^{\alpha}=-\frac{i}{42}\left(\Gamma^{c d}\right)^{\alpha \beta} \hat{D}_{\beta} H_{a b c d}
$$

and that there are no further independent components of $H$ at this level,

$$
\hat{D}_{\alpha} H_{a b c d}=-6 i\left(\Gamma_{[a b}\right)_{\alpha}{ }^{\beta} \hat{T}_{c d] \beta} .
$$

The dimension three-halves curvature is given by

$$
R_{\alpha b, c d}^{\prime}=-\frac{i}{2}\left(\Gamma_{b} \hat{T}_{c d}-\Gamma_{c} \hat{T}_{d b}+\Gamma_{d} \hat{T}_{c b}\right)_{\alpha}
$$

where

$$
\left(\Gamma_{b} \hat{T}_{c d}\right)_{\alpha}:=\left(\Gamma_{b}\right)_{\alpha}^{\beta} \hat{T}_{c d \beta} .
$$

We also find the following torsion constraint

$$
\left(\Gamma^{a b c}\right)_{\alpha}^{\beta} \hat{T}_{b c \beta}=0
$$

The leading component of this equation is the field equation for the gravitino.

At dimension two one shows that the curvature tensor is expressed as a second spinorial derivative of $H$ and that this is the only independent component of $H$ at this level. One also finds the equations of motion for the graviton

$$
R_{a b}^{\prime}-\frac{1}{2} \eta_{a b} R^{\prime}=-\frac{1}{48}\left(4 H_{a c d e} H_{b}^{c d e}-\frac{1}{2} \eta_{a b} H_{c d e f} H^{c d e f}\right)
$$

and

$$
\hat{D}^{a} H_{a b c d}=\frac{1}{36.48} \epsilon_{b c d e_{1} \ldots e_{8}} H^{e_{1} \ldots e_{4}} H^{e_{5} \ldots e_{8}}
$$

To complete the proof it is necessary to show that the four-index field $H$ can be derived from a three-form potential, in which case (18) will be the equation of motion for this field. Given the above results one may construct a superspace four-form $H_{4}$ with Weyl weight -6 which is covariantly closed,

$$
\hat{D} H_{4}:=d H_{4}+6 H_{4} \wedge K=0
$$

and which has non-vanishing components $H_{a b c d}$ and

$$
H_{\alpha \beta c d}=-i\left(\Gamma_{c d}\right)_{\alpha \beta}
$$

The proof that $H_{4}$ defined as above satisfies the Bianchi identity (19) is essentially the same as it is in the absence of the scale connection, and will not be repeated here. Since the curvature associated with $K$ vanishes we can deduce the existence of a three-from potential $B_{3}$, also of Weyl weight -6 , such that

$$
H=\hat{D} B_{3} .
$$

Hence the proof is complete. Since the equations of motion are Weyl invariant and since the scale curvature $G$ vanishes it follows that, if $H^{1}(M)=0, K$ can be transformed to zero so that 
we recover the standard equations of motion of eleven-dimensional supergravity in superspace form.

As in the standard on-shell formalism it is possible to construct a seven-form $H_{7}$ [7] which, in this case, has Weyl weight -12 and which satisfies

$$
\hat{D} H_{7}=\frac{1}{2}\left(H_{4}\right)^{2} \text {. }
$$

Its non-vanishing components are

$$
\begin{aligned}
H_{\alpha \beta a b c d e} & =-i\left(\Gamma_{\text {abcde }}\right)_{\alpha \beta} \\
H_{\text {abcdefg }} & =\frac{1}{4 !} \epsilon_{\text {abcdefghijk }} H^{h i j k} .
\end{aligned}
$$

It can be written in terms of potentials as

$$
H_{7}=\hat{D} B_{6}+\frac{1}{2} B_{3} \wedge H_{4}
$$

We shall now rewrite the above equations in Lorentzian superspace by reducing the structure group to $\operatorname{Spin}(1,10)$. This can be accomplished by simply taking the connection to be $\Omega^{\prime}$ so that the Weyl connection $K$ now appears in the torsion, that is,

$$
\begin{aligned}
& \hat{T}_{A B}{ }^{c}=T_{A B}^{\prime c}+2\left(K_{A} \delta_{B}{ }^{c}-(-1)^{A B} K_{B} \delta_{A}{ }^{c}\right) \\
& \hat{T}_{A B}{ }^{\gamma}=T_{A B}^{\prime}+\left(K_{A} \delta_{B}^{\gamma}-(-1)^{A B} K_{B} \delta_{A}^{\gamma}\right)
\end{aligned}
$$

where $T^{\prime}$ is the torsion constructed using only $\Omega^{\prime}$. However, this torsion does not satisfy the standard constraint that its dimension one component with purely vectorial indices vanish. In order to achieve this it is necessary to make a further redefinition of the purely vectorial part of the connection. It is simpler to combine this redefinition with the reduction of the structure group in one step. We therefore set

$$
\begin{aligned}
\hat{\Omega}_{\alpha \beta}{ }^{\gamma} & =\Omega_{\alpha \beta}{ }^{\gamma}+K_{\alpha} \delta_{\beta}{ }^{\gamma} \\
\hat{\Omega}_{\alpha b}{ }^{c} & =\Omega_{\alpha b}{ }^{c}+2 K_{\alpha} \delta_{b}{ }^{c} \\
\hat{\Omega}_{a \beta}{ }^{\gamma} & =\Omega_{a \beta}{ }^{\gamma}+K_{a} \delta_{\beta}{ }^{\gamma}-\left(\Gamma_{a}{ }^{b}\right)_{\beta}{ }^{\gamma} K_{b} \\
\hat{\Omega}_{a b}{ }^{c} & =\Omega_{a b}{ }^{c}+2\left(K_{a} \delta_{b}{ }^{c}+\delta_{a}{ }^{c} K_{b}-\eta_{a b} K^{c}\right) .
\end{aligned}
$$

The components of the new Lorentzian torsion are, at dimension zero,

$$
T_{\alpha \beta}^{c}=-i\left(\Gamma^{c}\right)_{\alpha \beta}
$$

at dimension one-half,

$$
T_{\alpha b}^{c}=-2 \delta_{b}^{c} K_{\alpha} ; \quad T_{\alpha \beta}^{\gamma}=-2 \delta_{(\alpha}{ }^{\gamma} K_{\beta)},
$$

and, at dimension one,

$$
T_{a \beta}^{\gamma}=-\frac{1}{36}\left(\left(\Gamma^{b c d}\right)_{\beta}^{\gamma} H_{a b c d}+\frac{1}{8}\left(\Gamma_{a b c d e}\right)_{\beta}^{\gamma} H^{b c d e}\right)-\left(\Gamma^{b} \Gamma_{a}\right)_{\beta}^{\gamma} K_{b},
$$


as well as $T_{a b}{ }^{c}=0$. The dimension three-halves torsion, since it does not involve a connection, is unchanged. For the curvature one has

$$
\begin{aligned}
R_{\alpha \beta, c d} & =R_{\alpha \beta, c d}^{\prime}+4 i K_{[c}\left(\Gamma_{d]}\right)_{\alpha \beta} \\
R_{\alpha b, c d} & =R_{\alpha b, c d}^{\prime}-4 \eta_{b[c} D_{\alpha} K_{d]}+8 \eta_{b[c} K_{d]} \\
R_{a b}{ }^{c d} & =R_{a b}^{\prime}{ }^{c d}+8 \delta_{[a}^{[c} D_{b]} K^{d]}-16 \delta_{[a}^{[c} K_{b]} K^{d]}-8 \delta_{[a}{ }^{c} \delta_{b]}{ }^{d} K^{2},
\end{aligned}
$$

where $R^{\prime}$ is the curvature in the original superspace and $K^{2}:=K^{a} K_{a}$. From the last of these equations one can compute the new Ricci tensor,

$$
R_{a b}=R_{a b}^{\prime}-18 D_{a} K_{b}-2 \eta_{a b} D^{c} K_{c}+36\left(K_{a} K_{b}-\eta_{a b} K^{2}\right) .
$$

The one-form $K=E^{\alpha} K_{\alpha}+E^{a} K_{a}$ is of course still closed. If it is exact it can be written as $d S$ for some $S$, and by making an appropriate super-Weyl transformation all the terms involving $K$ can be removed leaving the standard on-shell superspace again.

In summary, we have shown that the equations of motion of eleven-dimensional supergravity are implied by the standard constraint on the dimension zero torsion, at least if $M$ is simply connected. The proof of this assertion is simplified by the use of Weyl superspace, although it is not essential to introduce a scale connection. However, in Lorentzian superspace one has to prove that $K$ is closed and recognise that it can be removed by a super-Weyl transformation.

The formalism given here suggests a slight generalisation of standard eleven-dimensional supergravity when $M$ is not simply connected. For example, one might take $M$ to have the form $M^{10 \mid 32} \times S^{1}$ with $K \sim m d y$, where $y$ is the $S^{1}$ coordinate, thereby introducing a mass into the theory. This possibility is discussed elsewhere [8].

We conclude with some brief comments on a recent paper [9] by Nishino and Gates in which it is claimed that an off-shell extension of eleven-dimensional supergravity can be constructed in superspace involving a dimension one-half superfield. The authors use a Lorentzian structure group and include a closed four-form $H$ (called $F$ in [9]) from the beginning. In their notation, the basic dimension zero constraints are taken to be

$$
T_{\alpha \beta}^{c}=i\left(\Gamma^{c}\right)_{\alpha \beta} ; \quad H_{\alpha \beta c d}=\frac{1}{2}\left(\Gamma_{c d}\right)_{\alpha \beta}
$$

The dimension one-half torsion components are

$$
\begin{aligned}
T_{\alpha \beta}{ }^{\gamma} & =-8\left(\Gamma^{a}\right)_{\alpha \beta}\left(\Gamma_{a}\right)^{\gamma \delta} J_{\delta} \\
T_{\alpha b}{ }^{c} & =8\left(\Gamma^{c} \Gamma_{b}\right)_{\alpha}{ }^{\beta} J_{\beta}
\end{aligned}
$$

and the dimension one-half component of $H$ is

$$
H_{a b c \delta}=12 i\left(\Gamma_{a b c}\right)_{\delta}^{\epsilon} J_{\epsilon}
$$

while the components of $H$ with negative dimensions, $H_{\alpha \beta \gamma \delta}$ and $H_{\alpha \beta \gamma d}$, are assumed to vanish. The field $J_{\alpha}$ is the new auxiliary spinor superfield. However, it is not difficult to see that it can be removed from the dimension one-half tensors by a field redefinition of the form

$$
E_{a} \rightarrow E_{a}^{\prime}=E_{a}+\Lambda_{a}{ }^{\alpha} E_{\alpha} ; \quad E_{\alpha}^{\prime}=E_{\alpha}
$$

which amounts to a change of choice of even tangent bundle. The connection form is unchanged although the components of the vectorial part of the connection will change as a consequence of the above change of basis. In terms of differential forms one has

$$
E^{\prime a}=E^{a} ; \quad E^{\prime \alpha}=E^{\alpha}-E^{a} \Lambda_{a}^{\alpha}
$$


so that the torsion two forms change by

$$
\begin{aligned}
& T^{\prime a}=T^{a} \\
& T^{\prime \alpha}=T^{\alpha}-T^{a} \Lambda_{a}{ }^{\alpha}-E^{a} D \Lambda_{a}{ }^{\alpha}
\end{aligned}
$$

It is straightforward to compute the new torsion components as well as the change in the components of $H$. At dimension zero (and less) there is no change, and at dimension one-half one finds

$$
\begin{aligned}
T_{\alpha b}^{\prime c} & =T_{\alpha b}{ }^{c}-\Lambda_{b}{ }^{\beta} T_{\alpha \beta}{ }^{c} \\
T_{\alpha \beta}^{\prime}{ }^{\gamma} & =T_{\alpha \beta}{ }^{\gamma}-T_{\alpha \beta}{ }^{c} \Lambda_{c}^{\gamma} \\
H_{a b c \delta}^{\prime} & =H_{a b c \delta}+3 \Lambda_{[a}^{\gamma} H_{b c] \gamma \delta}
\end{aligned}
$$

If one makes the choice

$$
\Lambda_{a}^{\alpha}=8 i\left(\Gamma_{a}\right)^{\alpha \beta} J_{\beta}
$$

it is easy to see that the new dimension one-half components of the torsion and $H$ are zero. There are more complicated changes induced at dimension one and higher, but whatever they are we know from the results of [6, 7], or from a simple adaptation of the argument given earlier in this paper, that the equations of motion of eleven-dimensional supergravity will result. Hence the formalism of Nishino and Gates is strictly equivalent to standard on-shell supergravity in eleven dimensions. It differs from that of the current paper in that, even if spacetime is topologically trivial, the transformations required to recover standard on-shell superspace are not the same. In the Nishino-Gates approach this is accomplished by the above redefinition of $E_{a}$ whereas in our approach one needs to use a super-Weyl transformation. It is only after this transformation has been made that one finds that $H$ is closed in the usual sense.

A consequence of the above discussion is that the standard dimension zero constraint on the torsion tensor needs to be amended in order to incorporate the higher order corrections to supergravity which one would expect to arise in $M$-theory.

\section{Appendix}

In this appendix we give a few more details of the algebraic steps involved in the proof of the main result in the text. We suppress the hats throughout the appendix as all quantities are taken to be in the Weyl superspace.

There are two main computations to perform, at dimension one-half and at dimension one. At dimension one-half one is free to make redefinitions of the form

$$
E_{a} \rightarrow E_{a}+\Lambda_{a}^{\alpha} E_{\alpha}
$$

corresponding to making a choice of $B$, and one can impose further constraints in order to solve algebraically for the dimension one-half part of the connection. Using these freedoms, but without fixing the scale connection, it is not difficult to see that one can bring $T_{\alpha b}{ }^{c}$ to the form

$$
T_{\alpha b, c}=\tilde{T}_{\alpha b c}
$$

where the tensor on the right-hand side is symmetric and traceless on its vector indices and gamma-traceless. This is an irreducible representation of $\operatorname{Spin}(1,10)$ which does not occur in 
the decomposition of the other dimension one-half torsion $T_{\alpha \beta}{ }^{\gamma}$ into irreducibles. The dimension one-half Bianchi identity is, when $T_{\alpha \beta}^{c}=-i\left(\Gamma^{c}\right)_{\alpha \beta}$,

$$
T_{(\alpha \beta}{ }^{E} T_{|E| \gamma)}{ }^{d}=0 .
$$

This must be satisfied by the $T_{\alpha b}{ }^{c}$ of (46), but it is not difficult to see that this is not possible, and so we must have

$$
T_{\alpha b}{ }^{c}=0 .
$$

The Bianchi identity (47) then simplifies to

$$
T_{\alpha \beta}^{\epsilon}\left(\Gamma^{d}\right)_{\gamma) \epsilon}=0
$$

The remaining dimension one-half torsion is decomposed into irreducibles as follows

$$
T_{\alpha \beta}^{\gamma}=\sum_{n=1,2,5}\left(\Gamma^{a_{1} \ldots a_{n}}\right)_{\alpha \beta} \Psi_{a_{1} \ldots a_{n}}^{\gamma}
$$

with each $\Psi$ being a sum of gamma-traceless antisymmetric tensor-spinors with up to $n$ indices, for example,

$$
\Psi_{a b}=\psi_{a b}+\Gamma_{[a} \psi_{b]}+\Gamma_{a b} \psi
$$

where each of the $\psi^{\prime}$ s is irreducible. One thus has one each of such fields with three, four or five indices, two fields with two indices and three fields with zero and one index. Examining the Bianchi identity (49) for each of these representations in turn one finds that they all vanish except for the zero-index representations, of which only one is independent. The result of all this is that, making use of the completeness relation for symmetric gamma-matrices, one can write $T_{\alpha \beta}{ }^{\gamma}$ in the form

$$
T_{\alpha \beta}^{\gamma}=16 \delta_{(\alpha}^{\gamma} \Lambda_{\beta)}+6\left(\Gamma^{a}\right)_{\alpha \beta}\left(\Gamma_{a}\right)^{\gamma \delta} \Lambda_{\delta}-\left(\Gamma^{a b}\right)_{\alpha \beta}\left(\Gamma_{a b}\right)^{\gamma \delta} \Lambda_{\delta}
$$

This form for $T_{\alpha \beta}{ }^{\gamma}$ was derived in [7], but there is a difference here, namely that we still have the freedom to impose a constraint corresponding to the dimension one-half scale connection. Using this freedom one can impose $\Lambda=0$ so that all dimension one-half torsion components vanish as required. This final redefinition must be accompanied by further redefinitions of the Lorentzian part of the connection and of $E_{a}$. The required redefinition is

$$
\begin{aligned}
\Omega_{\alpha, b}{ }^{c} & \rightarrow \Omega_{a, b}{ }^{c}+\left(\Gamma_{b}{ }^{c}\right)_{\alpha}{ }^{\beta} \Lambda_{\beta}+\delta_{b}{ }^{c} \Lambda_{\alpha} \\
E_{a} & \rightarrow E_{a}+i\left(\Gamma_{a}\right)^{\alpha \beta} \Lambda_{\alpha} E_{\beta}
\end{aligned}
$$

Implementing this one finally arrives at

$$
T_{\alpha \beta}^{\gamma}=T_{\alpha b}^{c}=0
$$

We now turn to the dimension one analysis. There are two Bianchi identities:

$$
R_{\alpha \beta, c d}+2 \eta_{c d} G_{\alpha \beta}=-2 i T_{c(\alpha}^{\gamma}\left(\Gamma_{d}\right)_{\beta) \gamma}
$$

and

$$
R_{(\alpha \beta, \gamma)}{ }^{\delta}+\delta_{(\alpha}{ }^{\delta} G_{\beta \gamma)}=-i\left(\Gamma^{c}\right)_{(\alpha \beta} T_{c \gamma)}{ }^{\delta}
$$

The dimension one torsion may be written in the form

$$
T_{a \beta}^{\gamma}=\sum_{I=0}^{I=5}\left(\Gamma^{I}\right)_{\beta}{ }^{\gamma} T_{a, I}
$$


where the sum runs over the antisymmetrised $\Gamma$-matrices up to rank five. Each $T_{a, I}$ decomposes into three irreducible representations (except for $I=0$ ),

$$
T_{a, b_{1} \ldots b_{n}}=T_{a b_{1} \ldots b_{n}}+\eta_{a\left[b_{1}\right.} T_{\left.b_{2} \ldots b_{n}\right]}+\tilde{T}_{a, b_{1} \ldots b_{n}}
$$

The first two of these are totally antisymmetric while the third is traceless with vanishing totally antisymmetric part. $G_{\alpha \beta}$ can be written

$$
G_{\alpha \beta}=\left(\Gamma^{a b}\right)_{\alpha \beta} G_{a b}+\left(\Gamma^{a b c d e}\right)_{\alpha \beta} G_{a b c d e}
$$

since we may impose $\left(\Gamma_{a}\right)^{\alpha \beta} G_{\alpha \beta}=0$ as a conventional constraint corresponding to $K_{a}$. (Note that $G_{a b}$ here has dimension one and is not the same as the dimension two component of the two-form G.) Substituting these expressions in the symmetric part (on $c, d$ ) of the Bianchi identity (55), one finds a number of constraints on the tensors $T_{a, I}$. It is not difficult to verify that all of the $\tilde{T}$ tensors must vanish and that there are further relations between the remaining antisymmetric tensors. The result is

$$
\begin{aligned}
T_{a} & =0 \\
T_{a, b} & =\eta_{a b} T-2 i G_{a b} \\
T_{a, b c} & =T_{a b c} \\
T_{a, b c d} & =T_{a b c d}+i \eta_{a[b} G_{c d]} \\
T_{a, b c d e} & =5 i G_{a b c d e}+\eta_{a[b} T_{c d e]}^{\prime} \\
T_{a, b c d e f} & =\eta_{a[b} T_{c d e f]}^{\prime} \\
T_{a, b c d e f g} & =-i \eta_{a[b} G_{c d e f g]}
\end{aligned}
$$

where in the last line we have rewritten part of $T_{a, b c d e f}$ in the form $T_{a, b c d e f g}$ by dualising on the last five indices. One may now use the antisymmetric part of (55) to find the Lorentz curvature in terms of these antisymmetric tensors. Finally one substitutes these expressions into the Bianchi identity (56) and finds that they all vanish except those with four indices and that there is only one independent one of these. We thus arrive at the result (7) quoted in the text.

Acknowledgement. I thank Jim Gates for interesting discussions.

\section{References}

[1] E. Cremmer, B. Julia and J. Scherk, Supergravity theory in 11 dimensions, Phys. Lett. 76B (1978) 409.

[2] E. Cremmer and S. Ferrara, Formulation of 11-dimensional supergravity in superspace, Phys. Lett. 91B (1980) 61.

[3] L. Brink and P.S. Howe, Eleven dimensional supergravity on the mass shell in superspace, Phys. Lett. 91B (1980) 384.

[4] E. Bergshoeff, E. Sezgin and P.K. Townsend, Supermembranes and eleven-dimensional supergravity, Phys. Lett. 189B (1987) 75. 
[5] M.J. Duff, P.S. Howe, T. Inami and K.S. Stelle, Superstrings in D $=10$ from supermembranes in $D=11$.

[6] P.S. Howe, Pure spinors, function superspaces and supergravity theories in ten and eleven dimensions, Phys. Lett. B273 (1991), 90.

[7] A. Candiello and K. Lechner, Duality in supergravity theories, Nucl. Phys. B412 (1994), 479 .

[8] P.S. Howe, N.D. Lambert and P.C. West, A new massive type IIA supergravity from compactification KCL-TH-97-46, hep-th/9707139.

[9] H. Nishino and S.J. Gates, Jr. Towards an off-shell $11 D$ supergravity limit of $M$-theory, Phys. Lett. B388 (1996) 504. 